УДК 517.9

\title{
A Priori Estimates of the Adjoint Problem Describing the Slow Flow of a Binary Mixture and a Fluid in a Channel
}

\author{
Victor K. Andreev* \\ Marina V. Efimova ${ }^{\dagger}$ \\ Institute of Computational Modeling SB RAS \\ Akademgorodok, 50/44, Krasnoyarsk, 660036 \\ Siberian Federal University \\ Svobodny, 79, Krasnoyarsk, 660041
}

Russia

Received 21.03.2018, received in revised form 08.04.2018, accepted 25.06.2018

We obtain a priori estimates of the solution in the uniform metric for a linear conjugate initial-boundary inverse problem describing the joint motion of a binary mixture and a viscous heat-conducting liquid in a plane channel. With their help, it is established that the solution of the non-stationary problem with time growth tends to a stationary solution according to the exponential law when the temperature on the channel walls stabilizes with time.

Keywords: conjugate problem, inverse problem, a priori estimates, asymptotic behavior.

DOI: 10.17516/1997-1397-2018-11-4-482-493.

\section{Problem formulation}

Many natural and anthropogenic phenomena are described by models of thermal convection. The thermoconcentration flow occurs in inhomogeneously heated multiphase systems with an interface between phases or with a free surface between the liquid and the gas. The role of interfacial convection is great both on small scales, where volumetric effects, such as buoyancy, are insignificant, and under conditions of weightlessness, where the gravitational mechanisms of convective motion are weakened or absent [1-3].

We consider two layers of immiscible liquids between horizontal flat plates. The $X$ axis is directed horizontally, the $Y$ axis is vertically upward. Equations of rigid boundaries are $y=0, y=l_{2}$. The equation of the interphase surface is $y=l_{1}(x, t)$. The two-dimensional convective fluid flows are described by the Navier-Stokes equations in the Oberbeck-Boussinesq approximation. We introduce the dimensionless independent variables $\xi=x / l_{10}, \eta=y / l_{10}$, $\tau=\nu_{1} t / l_{10}^{2}, \mathbf{u}_{j}^{*}=\rho_{10} \nu_{1} \mathbf{u}_{j}\left(\mathfrak{x}_{1} \triangle \theta\right)^{(-1)}$ is the characteristic velocity, $P_{j}^{*}=l_{10} P_{j}\left(\mathfrak{l}_{1} \triangle \theta\right)^{(-1)}$ is the modified pressure, $\theta_{j}^{*}=\theta_{j} / \triangle \theta$ is the characteristic temperature. We can take $\triangle \theta=l_{10}^{2} \triangle A$, where $\triangle A=\max _{t \geqslant 0}\left|A_{20}(t)-A_{10}(t)\right|>0$. If $A_{20}(t)=A_{10}(t)$, then $\triangle A=\max _{j} \max _{y}\left|A_{j 0}(y)\right|>0$. $C^{*}=\beta_{1}^{c} l_{10}^{2} C\left(\beta_{1}^{\theta} \triangle \theta\right)^{(-1)}$ is the characteristic concentration of the light component; here $\nu_{1}$ is the constant kinematic viscosity, $\triangle \theta$ is the characteristic temperature drop, $l_{10}=\max \left|l_{1}(x, 0)\right|$. Then the Oberbeck-Boussinesq equations are written in the following dimensionless form (the

\footnotetext{
*andr@icm.krasn.ru

†efmavi@mail.ru

(c) Siberian Federal University. All rights reserved
} 
sign asterisk is omitted, $u_{j}, v_{j}$ are the velocity components)

$$
\begin{gathered}
u_{j \tau}+M\left(u_{j} u_{j \xi}+v_{j} u_{j \eta}\right)+\frac{\rho_{j 0}}{\rho_{10}} P_{j \xi}=\frac{\nu_{j}}{\nu_{1}}\left(u_{j \xi \xi}+u_{j \eta \eta}\right), \\
v_{j \tau}+M\left(u_{j} v_{j \xi}+v_{j} v_{j \eta}\right)+\frac{\rho_{j 0}}{\rho_{10}} P_{j \eta}=\frac{\nu_{j}}{\nu_{1}}\left(v_{j \xi \xi}+v_{j \eta \eta}\right)+G_{j}\left(\theta_{j}+\frac{\beta_{j}^{c}}{\beta_{1}^{c}} C\right), \\
\theta_{j \tau}+M\left(u_{j} \theta_{j \xi}+v \theta_{j \eta}\right)=\frac{\chi_{j}}{\nu_{1}}\left(\theta_{j \xi \xi}+\theta_{j \eta \eta}\right), \\
C_{\tau}+M\left(u_{1} C_{\xi}+v_{1} C_{\eta}\right)=\frac{1}{S}\left(C_{\xi \xi}+C_{\eta \eta}-\psi\left(\theta_{1 \xi \xi}+\theta_{1 \eta \eta}\right)\right), \\
u_{j \xi}+v_{j \eta}=0 .
\end{gathered}
$$

The dimensionless parameters arise in the problem: $M=x_{1} \triangle \theta l_{10} /\left(\rho_{10} \nu_{1}^{2}\right)$ is the Marangoni thermal number, $P r_{j}=\nu_{j} / \chi_{j}$ is the Prandtl number, $S=\nu_{1} / D$ is the Schmidt number, $\psi=-\alpha \beta_{1}^{c} / \beta_{1}^{\theta}$ is the separation parameter, $\nu=\nu_{2} / \nu_{1}$ is the kinematic viscosity ratio, $D$ is the constant diffusion coefficient, $\alpha D$ is the thermal diffusion coefficient Soret, $G_{j}=g \beta_{1}^{\theta} \rho_{10} / æ_{1}$ is the Grashof parameters; $\beta_{j}^{\theta}, \beta_{1}^{c}$ are the constant coefficients of the thermal and concentration expansion of the media, $\beta_{2}^{c}=0 ; W e=\sigma^{0} / \mathfrak{x}_{1} \triangle \theta$ is the Weber number, $\omega=\mathfrak{x}_{2} \beta_{1}^{\theta} / \mathfrak{x}_{1} \beta_{1}^{c}$ and $B o=\left(\rho_{2}-\rho_{1}\right) g l_{2}^{2} / \sigma^{0}$ is the Bond number. It is assumed that $B o \ll 1, W e \gg 1$ [4], then $l_{1 \xi \xi}=0$ and the interface can be a straight line only. We assume additionally that the motion in the layers is creeping $(M \ll 1)$. In this case the problem (1) becomes linear.

Let us assume that solution of linear systems (1) has the form

$$
\begin{gathered}
u_{j}=U_{j}(\eta, \tau) \xi, \quad v_{j}=V_{j}(\eta, \tau) \\
\theta_{j}=A_{j}(\eta, \tau) \xi^{2}+B_{j}(\eta, \tau), \quad C=H(\eta, \tau) \xi^{2}+E(\eta, \tau) \\
P_{j}=P_{j}(\xi, \eta, \tau) .
\end{gathered}
$$

Substitution (2) in system (1) reduces it to the following equations

$$
\begin{gathered}
A_{j \tau}=\frac{\chi_{j}}{\nu_{1}} A_{j \eta \eta}, \quad B_{j \tau}=\frac{\chi_{j}}{\nu_{1}}\left(2 A_{j}+B_{j \eta \eta}\right) \\
H_{\tau}=\frac{1}{S}\left(H_{\eta \eta}-\psi A_{1 \eta \eta}\right), \quad E_{\tau}=\frac{1}{S}\left(2 H+E_{\eta \eta}-\psi\left(2 A_{1}+B_{1 \eta \eta}\right)\right) \\
\frac{\nu_{j}}{\nu_{1}} U_{j \eta \eta}-U_{j \tau}=2 G_{j} \int_{\Omega_{j}}\left(A_{j}+\frac{\beta_{j}^{c}}{\beta_{1}^{c}} H\right) d \eta+R_{j}(\tau) \\
V_{j \eta}=-U_{j}, \quad\left(\Omega_{1}=(0, \eta), \quad \Omega_{2}=(1, \eta)\right) .
\end{gathered}
$$

The functions $P_{j}(\xi, \eta, \tau)$ have representations

$$
\frac{\rho_{10}}{\rho_{j 0}} P_{j}=\left(\frac{\nu_{j}}{\nu_{1}} U_{j \eta \eta}-U_{j \tau}\right) \frac{\xi^{2}}{2}+h_{j}(\tau, \eta), \quad h_{j \eta}=\frac{\nu_{j}}{\nu_{1}} V_{j \eta \eta}+G_{j} B_{j}+\frac{\beta_{j}^{c}}{\beta_{1}^{c}} G_{1} E-V_{j \tau} .
$$

Boundary conditions on solid walls are

$$
\begin{gathered}
U_{1}(0, \tau)=0, \quad U_{2}(l, \tau)=0, \quad A_{1}(0, \tau)=A_{10}(\tau), \quad A_{2}(l, \tau)=A_{20}(\tau) \\
B_{1}(0, \tau)=B_{10}(\tau), \quad B_{2}(l, \tau)=B_{20}(\tau) \\
H_{\eta}(0, \tau)-\psi A_{1 \eta}(0, \tau)=0 ; \quad E_{\tau}(0, \tau)-\psi B_{1 \eta}(0, \tau)=0
\end{gathered}
$$

Conditions on the interface for $\eta=1[4]$ are

$$
\begin{gathered}
U_{1}=U_{2}, \quad \mu U_{2 \eta}-U_{1 \eta}=-2 A_{1}-2 \omega H, \quad A_{1}=A_{2}, \quad A_{1 \eta}=k A_{2 \eta} \\
B_{1}=B_{2}, \quad B_{1 \eta}=k B_{2 \eta}, \quad H_{\eta}-\psi A_{1 \eta}=0, \quad E_{\eta}-\psi B_{1 \eta}=0
\end{gathered}
$$


In additionally

$$
\int_{0}^{1} U_{1}(z, \tau) d z=0, \quad \int_{1}^{l} U_{2}(z, \tau) d z=0 .
$$

Here we have introduced the notation $\rho=\rho_{20} / \rho_{10}, l=l_{2} / l_{10}>1, \mu=\rho \nu, k=k_{2} / k_{1}$. We supplement the problem with the initial conditions

$$
\begin{gathered}
U_{j}(y, 0)=0, \quad V_{j}(y, 0)=0, \quad A_{j}(y, 0)=A_{j}^{0}(y), \\
B_{j}(y, 0)=B_{j}^{0}(y), \quad H(y, 0)=H^{0}(y), \quad E(y, 0)=E^{0}(y) .
\end{gathered}
$$

The initial conditions for the velocities are taken to be zero, since we are interested in motion under the action of surface forces and buoyancy forces.

The integral conditions (4) are consequences of the immobility of the $\eta=1$ interface and the mass conservation equations, since from the last equation (3)

$$
V_{1}=-\int_{0}^{\eta} U_{1}(z, \tau) d z, \quad V_{2}=-\int_{\eta}^{l} U_{2}(z, \tau) d z
$$

and $V_{1}(1, \tau)=V_{2}(1, \tau)=0$.

We note the peculiarity of the problem posed: it is inverse, since the functions $R_{j}(\tau)$ must be determined together with $A_{j}(\eta, \tau), B_{j}(\eta, \tau), U_{j}(\eta, \tau)$. First, we define the function A, then we determine $H$ and $U_{j}$, the functions $E, B_{j}$ do not affect the velocity field, and the vertical velocities $V_{j}$ in the layers are found from the equalities (5). By the representation (2), the temperature on the walls has a minimum at $\xi=0(x=0)$ for $A_{j 0}(\tau)>0$, or a maximum for $A_{j 0}(\tau)<0$, or they alternate. Due to the Marangoni effect, the liquid and mixture can move in different directions.

\section{A priori estimates of the function $A_{j}(\tau, \eta), H(\tau, \eta)$}

The problem for functions $A_{j}(\eta, \tau)$ is separated. It has the form

In addition,

$$
\begin{gathered}
A_{1 \tau}=\frac{1}{P r_{1}} A_{1 \eta \eta}, \quad 0<\eta<1, \quad \tau \in[0, T], \\
A_{2 \tau}=\frac{\nu}{P r_{2}} A_{2 \eta \eta}, \quad 1<\eta<l, \quad \tau \in[0, T] \\
A_{1}(1, \tau)=A_{2}(1, \tau), \quad A_{1 \eta}(1, \tau)=k A_{2 \eta}(1, \tau) \\
A_{1}(0, \tau)=A_{10}(\tau), \quad A_{2}(l, \tau)=A_{20}(\tau) .
\end{gathered}
$$

$$
A_{1}(\eta, 0)=A_{1}^{0}(\eta), \quad A_{2}(\eta, 0)=A_{2}^{0}(\eta),
$$

where $A_{j 0}(\tau), \tau \in[0, T]$ and $A_{1}^{0}(\eta), 0<\eta<1, A_{2}^{0}(\eta), 1<\eta<l$ are the known functions.

The matching conditions for solutions of problem (6)-(8) are satisfied:

$$
A_{1}^{0}(1)=A_{2}^{0}(1), \quad A_{1 \eta}^{0}(1)=k A_{2 \eta}^{0}(1), \quad A_{10}(0)=A_{1}^{0}(0), \quad A_{20}(l)=A_{2}^{0}(l) .
$$

We replace the unknown functions

$$
\begin{aligned}
& A_{1}(\eta, \tau)=\bar{A}_{1}(\eta, \tau)+A_{10}(\tau)(\eta-1)^{2}, \quad 0 \leqslant \eta \leqslant 1, \\
& A_{2}(\eta, \tau)=\bar{A}_{2}(\eta, \tau)+\frac{A_{20}(\tau)(\eta-1)^{2}}{(l-1)^{2}}, \quad 1 \leqslant \eta \leqslant l .
\end{aligned}
$$


Then the functions $\bar{A}_{j}(\eta, \tau)$ are solutions of the conjugate initial-boundary-value problem

$$
\begin{gathered}
\bar{A}_{1 \tau}=\frac{1}{P r_{1}} \bar{A}_{1 \eta \eta}+\frac{2 A_{10}(\tau)}{P r_{1}}-A_{10}^{\prime}(\tau)(\eta-1)^{2} \equiv \\
\equiv \frac{1}{P r_{1}} \bar{A}_{1 \eta \eta}+f_{1}(\eta, \tau), \quad 0<\eta<1, \tau \in[0, T] \\
\bar{A}_{2 \tau}=\frac{\nu}{P r_{2}} \bar{A}_{2 \eta \eta}+\frac{2 \nu A_{20}(\tau)}{(l-1)^{2} P r_{2}}-A_{20}^{\prime} \frac{(\tau)(\eta-1)^{2}}{(l-1)^{2}} \equiv \\
\equiv \frac{\nu}{P r_{2}} \bar{A}_{2 \eta \eta}+f_{2}(\eta, \tau), \quad 1<\eta<l, \tau \in[0, T] \\
\bar{A}_{1}(1, \tau)=\bar{A}_{2}(1, \tau), \quad \bar{A}_{1 \eta}(1, \tau)=k \bar{A}_{2 \eta}(1, \tau), \quad \bar{A}_{1}(0, \tau)=0, \quad \bar{A}_{2}(l, \tau)=0 \\
\bar{A}_{1}(\eta, 0)=A_{1}^{0}(\eta)-A_{1}(0)(\eta-1)^{2} \equiv \bar{A}_{1}^{0}(\eta) \\
\bar{A}_{2}(\eta, 0)=A_{2}^{0}(\eta)-\frac{A_{2}(0)(\eta-1)^{2}}{(l-1)^{2}} \equiv \bar{A}_{2}^{0}(\eta)
\end{gathered}
$$

The prime denotes differentiation with respect to $\tau$ in the right-hand sides of equations (10), (11).

We multiply equation (10) by $\operatorname{Pr}_{1} \bar{A}_{1}$, equation (11) by $k \nu^{-l} \operatorname{Pr}_{2} \bar{A}_{2}$, integrate them over the domains of definition and add the results. Taking into account the boundary conditions (12), we obtain the identity

$$
\begin{gathered}
\frac{d}{d \tau} W(\tau)+\int_{0}^{1} \bar{A}_{1 \eta}^{2} d \eta+k \int_{1}^{l} \bar{A}_{2 \eta}^{2} d \eta=\operatorname{Pr}_{1} \int_{0}^{1} f_{1} \bar{A}_{1} d \eta+k \nu^{-1} \operatorname{Pr}_{2} \int_{1}^{l} f_{2} \bar{A}_{2} d \eta \\
W(\tau)=\frac{\operatorname{Pr}_{1}}{2} \int_{0}^{1} \bar{A}_{1}^{2}(\eta, \tau) d \eta+\frac{k P r_{2}}{2 \nu} \int_{1}^{l} \bar{A}_{2}^{2}(\eta, \tau) d \eta
\end{gathered}
$$

Since [5]

$$
\int_{0}^{1} \bar{A}_{1}^{2} d \eta+\int_{1}^{l} \bar{A}_{2}^{2} d \eta \leqslant M_{1}\left(\int_{0}^{1} \bar{A}_{1 \eta}^{2} d \eta+k \int_{1}^{l} \bar{A}_{2 \eta}^{2} d \eta\right)
$$

with a finite minimal positive constant $M_{1}$ depending on $k$ and $l$, then the left-hand side of (14) is greater than or equal to

$$
\frac{d W}{d \tau}+2 \delta W, \quad \delta=\frac{1}{M_{1}} \min \left(\frac{1}{P r_{1}}, \frac{\nu}{k P r_{2}}\right)
$$

The right-hand side of (14), using the Holder inequality, does not exceed

$$
\left[\left(2 \operatorname{Pr}_{1} \int_{0}^{1} f_{1}^{2} d \eta\right)^{1 / 2}+\left(2 k \nu^{-1} \operatorname{Pr}_{2} \int_{1}^{l} f_{2}^{2} d \eta\right)^{1 / 2}\right] \sqrt{W(\tau)} \equiv G(\tau) \sqrt{W(\tau)}
$$

From (16), (17) we obtain the inequality

$$
\begin{gathered}
W(\tau) \leqslant\left(\sqrt{W_{0}}+\frac{1}{2} \int_{0}^{\tau} G(\tau) e^{\delta t} d t\right)^{2} e^{-2 \delta \tau} \\
W_{0}=\frac{P r_{1}}{2} \int_{0}^{1}\left(\bar{A}_{1}^{0}(\eta)\right)^{2} d \eta+\frac{k P r_{2}}{2 \nu} \int_{1}^{l}\left(\bar{A}_{2}^{0}(\eta)\right)^{2} d \eta \\
-485-
\end{gathered}
$$


where the initial values $\bar{A}_{1}^{0}(\eta), \bar{A}_{2}^{0}(\eta)$ are defined by the equalities (13).

It turns out that one can obtain the estimates $\left|\bar{A}_{j}(\eta, \tau)\right|$. To do this, note that along with (14) there is also another identity for the problem (10)-(13)

$$
\begin{gathered}
\operatorname{Pr}_{1} \int_{0}^{1} \bar{A}_{1 \tau}^{2} d \eta+k \nu^{-1} \operatorname{Pr}_{2} \int_{1}^{l} \bar{A}_{2 \tau}^{2} d \eta+\frac{1}{2} \frac{d}{d \tau}\left[\int_{0}^{1} \bar{A}_{1 \eta}^{2} d \eta+k \int_{1}^{l} \bar{A}_{2 \eta}^{2} d \eta\right]= \\
=\operatorname{Pr}_{1} \int_{0}^{1} f_{1} \bar{A}_{1 \tau} d \eta+k \nu^{-1} \operatorname{Pr}_{2} \int_{1}^{l} f_{2} \bar{A}_{2 \tau} d \eta
\end{gathered}
$$

from which it follows that

$$
\begin{gathered}
\int_{0}^{1} \bar{A}_{1 \tau}^{2} d \eta+k \int_{1}^{l} \bar{A}_{2 \tau}^{2} d \eta \leqslant \int_{0}^{1}\left(\bar{A}_{1 \eta}^{0}\right)^{2} d \eta+k \int_{1}^{l}\left(\bar{A}_{2 \eta}^{0}\right)^{2} d \eta+ \\
+\operatorname{Pr}_{1} \int_{0}^{\tau} \int_{0}^{1} f_{1}^{2} d \eta d \tau+k \nu^{-1} \operatorname{Pr}_{2} \int_{0}^{\tau} \int_{1}^{l} f_{2}^{2} d \eta d \tau \equiv F(\tau)
\end{gathered}
$$

with $F(\tau)$ bounded on $[0, T]$. Since

$$
\bar{A}_{1}^{2}(\eta, \tau)=2 \int_{0}^{\eta} \bar{A}_{1}(\eta, \tau) \bar{A}_{1 \eta}(\eta, \tau) d \eta, \quad \bar{A}_{2}^{2}(\eta, \tau)=-2 \int_{\eta}^{l} \bar{A}_{2}(\eta, \tau) \bar{A}_{2 \eta}(\eta, \tau) d \eta,
$$

using the Hölder inequality, the definition of the function $W(\tau)(15)$, the estimate (19) and substitutions (9) we obtain

$$
\begin{gathered}
\left|A_{1}(\eta, \tau)\right| \leqslant\left|A_{10}(\tau)\right|+\left(\frac{8}{P r_{1}} W(\tau) F(\tau)\right)^{1 / 4} \\
\left|A_{2}(\eta, \tau)\right| \leqslant\left|A_{20}(\tau)\right|+\left(\frac{8 \nu}{k^{2} P r_{2}} W(\tau) F(\tau)\right)^{1 / 4}
\end{gathered}
$$

uniform in $\eta \in[0,1]$ and $\eta \in[1, l]$ respectively. In (20) the quantity $F(\tau)$ is given by formula (19) and $W(\tau)$ is estimated from above by the right-hand side of (18). Therefore, the quantities $\left|A_{j}(\eta, \tau)\right|$ are bounded for $\tau \in[0, T]$, if they are $A_{j 0}(\tau)$ and $A_{j 0}^{\prime}(\tau), j=1,2$.

Further, we need estimates of the derivatives $\eta$ uniform in $A_{j \tau}(\eta, \tau)$. To this end, we differentiate with respect to $\tau$ the equations (6) and the boundary conditions (7), assuming the existence of $A_{j 0}^{\prime \prime}(\tau)$. Then the problem for the functions $A_{j \tau}$ coincides exactly with the problem for $A_{j}$ with changed initial data $A_{1 \tau}(\eta, 0)=A_{1 \eta \eta}^{0} / P r_{1}, \quad A_{2 \tau}(\eta, 0)=\nu A_{2 \eta \eta}^{0} / P r_{2}, \quad$ and the right-hand sides of the last two boundary conditions $(7)$ are $A_{10}^{\prime}(\tau), A_{20}^{\prime}(\tau)$. Therefore, we obtain estimates of the form (20):

$$
\begin{gathered}
\left|A_{1 \tau}(\eta, \tau)\right| \leqslant\left|A_{10}^{\prime}(\tau)\right|+\left(\frac{8}{P r_{1}} W_{1}(\tau) F_{1}(\tau)\right)^{1 / 4}, \\
\left|A_{2 \tau}(\eta, \tau)\right| \leqslant\left|A_{20}^{\prime}(\tau)\right|+\left(\frac{8 \nu}{k^{2} P r_{2}} W_{1}(\tau) F_{1}(\tau)\right)^{1 / 4},
\end{gathered}
$$

where $W_{1}(\tau)$ satisfies the inequality $(18)$ with $A_{j 0}(\tau)$ replaced by $A_{j 0}^{\prime}(\tau), A_{j 0}^{\prime}(\tau)$ by $A_{j 0}^{\prime \prime}(\tau)$. A similar change must be made in the expression for $F(\tau)$ to obtain $F_{1}(\tau)$ (more precisely, in the functions $f_{j}(\eta, \tau)$ from $\left.(10),(11)\right)$. In addition, the initial data in $W_{10}$ and $F_{1}(\tau)$ should be replaced by $\operatorname{Pr}_{1}^{-1} A_{1 \eta \eta}^{0}-A_{10}^{\prime}(0)(\eta-1)^{2}$ and $\nu P r_{2}{ }^{-1} A_{2 \eta \eta}^{0}-A_{20}^{\prime}(0)(l-1)^{-2}(\eta-1)^{2}$, respectively. 
We introduce the substitution $H_{1}=H-\psi A_{1}$ to obtain an estimate of the functions $H(\eta, \tau)$. Then $H_{1}(\eta, \tau)$ is a solution of the initial boundary value problem

$$
\begin{gathered}
H_{1 \tau}=\frac{1}{S} H_{1 \eta \eta}-\psi A_{1 \tau}, \quad 0<\eta<1, \quad \tau \in[0, T] ; \\
H_{1}(\eta, 0)=H_{1}^{0}(\eta)-\psi A_{1}^{0}(\eta) \equiv H_{1}^{0}(\eta), \quad 0<\eta<1 ; \\
H_{1 \eta}(0, \tau)=0, \quad H_{1 \eta}(1, \tau)=0, \quad \tau \in[0, T] .
\end{gathered}
$$

The problem for the known $A_{1 \tau}(\eta, \tau)$ and $H_{1}^{0}(\eta)$ has the solution [6]

$$
\begin{gathered}
H_{1}(\eta, \tau)=\int_{0}^{1} H_{1}^{0}(\xi) G(\eta, \xi, \tau) d \xi-\psi \int_{0}^{\tau} \int_{0}^{1} A_{1 \mu}(\xi, \mu) G(\eta, \xi, \tau-\mu) d \xi d \mu \\
G(\eta, \xi, \tau)=1+2 \sum_{n=1}^{\infty} \cos n \pi \eta \cos n \pi \xi \exp \left(-\frac{n^{2} \pi^{2}}{S} \tau\right)= \\
=\frac{1}{2} \sqrt{\frac{S}{\pi \tau}} \sum_{n=-\infty}^{\infty}\left\{\exp \left[-\frac{S(\eta-\xi+2 n)^{2}}{4 \tau}\right]-\exp \left[-\frac{S(\eta+\xi+2 n)^{2}}{4 \tau}\right]\right\} .
\end{gathered}
$$

From the representation (23) and the boundedness of $A_{1 \mu}(\xi, \mu)$ (estimate (21)), it follows that $\left|H_{1}(\eta, \tau)\right|$ is bounded for all $\eta \in[0,1]$ and $\tau \in[0, T] .|H(\eta, \tau)|$ is also bounded because $H=H_{1}+\psi A_{1}$ and by (20). In addition, the derivative $H_{\tau}(\eta, \tau)$ is continuous and bounded for $\eta \in[0,1], \tau \in[0, T]$.

\section{A priori estimates of the functions $U_{j}(\tau, \eta)$ and $R_{j}(\tau)$}

We consider the problem for the definition of functions $U_{j}(\eta, t), R_{j}(\tau)$ :

$$
\begin{gathered}
U_{1 \eta \eta}-U_{1 \tau}=2 G_{1} \int_{0}^{\eta}\left(A_{1}(z, \tau)+H(z, \tau)\right) d z+R_{1}(\tau), \quad 0<\eta<1, \\
\nu U_{2 \eta \eta}-U_{2 \tau}=2 G_{2} \int_{1}^{\eta} A_{2}(z, \tau) d z+R_{2}(\tau), \quad 1<\eta<l, \\
U_{1}(\eta, 0)=0 \quad(0<\eta<1), \quad U_{2}(\eta, 0)=0 \quad(1<\eta<l), \\
U_{1}(1, \tau)=U_{2}(1, \tau), \quad \mu U_{2 \eta}(1, \tau)-U_{1 \eta}(1, \tau)=-2 A_{1}(1, \tau)-2 \omega H(1, \tau), \\
U_{1}(0, \tau)=U_{2}(l, \tau)=0, \\
\int_{0}^{1} U_{1}(z, \tau) d z=0, \quad \int_{1}^{l} U_{2}(z, \tau) d z=0 .
\end{gathered}
$$

We introduce the notations

$$
\begin{gathered}
F_{1}(\eta, \tau)=-2 G_{1} \int_{0}^{\eta}\left(A_{1}(z, \tau)+H(z, \tau)\right) d z, \quad 0<\eta<1, \quad \tau \in[0, T], \\
F_{2}(\eta, \tau)=-2 G_{2} \int_{1}^{\eta} A_{2}(z, \tau) d z, \quad 1<\eta<l, \quad \tau \in[0, T] \\
F_{3}(\tau)=-2 A_{1}(1, \tau)-2 \omega H(1, t), \quad \tau \in[0, T]
\end{gathered}
$$

$F_{1}, F_{2}, F_{3}$ are continuous and differentiable on their domains of definition by what has been proved above. 
Let's make a replacement

$$
U_{1}(\eta, \tau)=\bar{U}_{1}(\eta, \tau)-F_{3}(\tau)\left(2 \eta^{3}-3 \eta^{2}+\eta\right),
$$

then the second boundary condition (28) becomes homogeneous for the functions $\bar{U}_{1}(\eta, \tau)$, $U_{2}(\eta, \tau)$. The conditions (27)-(30) for these functions also remain homogeneous. Equation (25) for $\bar{U}_{1}(\eta, \tau)$ takes the form

$$
\bar{U}_{1 \eta \eta}-\bar{U}_{1 \eta}=R_{1}(\tau)-F_{1}(\eta, \tau)+6 F_{3}(\tau)(2 \eta-1)-F_{3}^{\prime}(\tau)\left(2 \eta^{3}-3 \eta^{2}+\eta\right) \equiv R_{1}(\tau)-\bar{F}_{1}(\eta, \tau) .
$$

In addition, the first initial condition (27) will change $\bar{U}_{1}(\eta, 0)=F_{3}(0)\left(2 \eta^{3}-3 \eta^{2}+\eta\right) \equiv$ $\bar{U}_{10}(\eta)$.

We multiply equation (33) by $\bar{U}_{1}$, (26) by $\rho U_{2}$, then integrate over $\eta$ and add the results. Using homogeneous boundary conditions (27)-(30) for $\bar{U}_{1}, U_{2}$, we obtain the identity

$$
\begin{gathered}
\frac{d E_{1}}{d \tau}+\int_{0}^{1} \bar{U}_{1 z}^{2} d z+\mu \int_{1}^{l} U_{2 z}^{2} d z=\int_{0}^{1} \bar{F}_{1} \bar{U}_{1} d z+\rho \int_{1}^{l} F_{2} U_{2} d z, \\
E_{1}(\tau)=\frac{1}{2} \int_{0}^{1} \bar{U}_{1}^{2}(z, \tau) d z+\frac{\rho}{2} \int_{1}^{l} U_{2}^{2}(z, \tau) d z .
\end{gathered}
$$

Since for $\bar{U}_{1}, U_{2}$ the following inequalities hold Friedrichs theorem, from (34) we obtain the inequality

$$
\begin{gathered}
\frac{d E_{1}}{d \tau}+2 \delta E_{1} \leqslant 2 G_{1}(\tau) \sqrt{E_{1}}, \\
\delta=2 \min \left(1, \frac{\mu}{\rho(l-1)^{2}}\right), \quad G_{1}(\tau)=\frac{1}{\sqrt{2}}\left[\left(\int_{0}^{1} \bar{F}_{1}^{2} d z\right)^{1 / 2}+\sqrt{\rho}\left(\int_{1}^{l} F_{2}^{2} d z\right)^{1 / 2}\right],
\end{gathered}
$$

whence the estimate

$$
E_{1}(\tau) \leqslant\left(\sqrt{E_{1}(0)}+\int_{0}^{\tau} G_{1}\left(\tau_{1}\right) e^{2 \delta \tau_{1}} d \tau_{1}\right)^{2} e^{-2 \delta \tau}, \quad E_{1}(0)=\frac{1}{2} \int_{0}^{1} \bar{U}_{10}^{2} d z .
$$

Hence the norm of the functions $\bar{U}_{1}(\eta, \tau), U_{2}(\eta, \tau)$ in the space $L_{2}$ is bounded for $\tau \in[0, T]$

$$
\int_{0}^{1} \bar{U}_{1}^{2} d z \leqslant 2 E_{1}(\tau), \quad \int_{1}^{l} U_{2}^{2} d z \leqslant \frac{2}{\rho} E_{1}(\tau)
$$

Similarly to (19) we have the inequality

$$
\int_{0}^{1} \bar{U}_{1 z}^{2} d z+\mu \int_{1}^{l} U_{2 z}^{2} d z \leqslant \int_{0}^{1} \bar{U}_{10 z}^{2}(z) d z+\int_{0}^{\tau} \int_{0}^{1} \bar{F}_{1}^{2} d z d \tau+\rho \int_{0}^{\tau} \int_{1}^{l} F_{2}^{2} d z d \tau \equiv Q(\tau) .
$$

Using (37), (38) and replacing (32), we obtain the estimate

$$
\left|U_{1}(\eta, \tau)\right| \leqslant\left(8 E_{1}(\tau) Q(\tau)\right)^{1 / 4}+6\left|F_{3}(\tau)\right|,
$$

uniform for $\eta \in[0,1], \tau \in[0, T]$. Similarly,

$$
\left|U_{2}(\eta, \tau)\right| \leqslant\left(\frac{8}{\rho \mu} E_{1}(\tau) Q(\tau)\right)^{1 / 4}
$$


To obtain the estimates $\left|R_{j}(\tau)\right|, \tau \in[0, T]$, it is necessary to estimate $\left|U_{j \tau}(\eta, \tau)\right|$. We differentiate equations $(25),(26)$ and conditions $(27)-(30)$ with respect to $\tau$. Taking into account the notation (31), we obtain a problem for $Y_{j}(\eta, \tau)=U_{j \tau}(\eta, \tau)$

$$
\begin{gathered}
Y_{1 \eta \eta}-Y_{1 \tau}=-F_{1 \tau}+R_{1 \tau}, \quad 0<\eta<1 ; \\
\nu Y_{2 \eta \eta}-Y_{2 \tau}=-F_{2 \tau}+R_{2 \tau}, \quad 1<\eta<l ; \\
Y_{1}(1, \tau)=Y_{2}(1, \tau), \quad \mu Y_{2 \eta}(1, \tau)-Y_{1 \eta}(1, \tau)=F_{3 \tau}, \\
Y_{1}(0, \eta)=0, \quad Y_{2}(l, \tau)=0, \\
\int_{0}^{1} Y_{1}(z, \tau) d z=0, \quad \int_{1}^{l} Y_{2}(z, \tau)=0 .
\end{gathered}
$$

The initial data for $\tau=0$ for equations (40) follow from (25), (26):

$$
Y_{1}(\eta, 0)=F_{1}(\eta, 0)-R_{1}(0) \equiv Y_{1}^{0}(\eta), \quad Y_{2}(\eta, 0)=F_{2}(\eta, 0)-R_{2}(0) \equiv Y_{2}^{0}(\eta)
$$

Integrating (25) with respect to $\eta$ from 0 to $1,(26)$ from 1 to $l$, we find the unknown quantities $R_{j}(0)$ with allowance for $(27),(29)$

$$
R_{1}(0)=\int_{0}^{1} F_{1}(z, 0) d z, \quad R_{2}(0)=\frac{1}{l-1} \int_{1}^{l} F_{2}(z, 0) d z
$$

For the initial boundary value problem (40)-(43), the identity

$$
\begin{gathered}
\frac{d E_{2}}{d \tau}+\int_{0}^{1} Y_{1 z}^{2} d z+\mu \int_{1}^{l} Y_{2 z}^{2} d z=\int_{0}^{1} F_{1 \tau} Y_{1} d z+\rho \int_{1}^{l} F_{2 \tau} Y_{2} d z-F_{3 \tau} Y_{1}(1, \tau) \\
E_{2}(\tau)=\frac{1}{2} \int_{0}^{1} Y_{1}^{2} d z+\frac{\rho}{2} \int_{1}^{l} Y_{2}^{2} d z
\end{gathered}
$$

The right-hand side of (44) does not exceed

$$
\frac{1}{2 \varepsilon_{1}} \int_{0}^{1} F_{1 \tau}^{2} d z+\frac{\varepsilon_{1}}{2} \int_{0}^{1} Y_{1}^{2} d z+\frac{\rho}{2 \varepsilon_{2}} \int_{1}^{l} F_{2 \tau}^{2} d z+\frac{\rho \varepsilon_{2}}{2} \int_{1}^{l} Y_{2}^{2} d z+\frac{1}{2} F_{3 \tau}^{2}+\frac{1}{2} \int_{0}^{1} Y_{1 z}^{2} d z
$$

for any $\varepsilon_{1}>0, \varepsilon_{2}>0$. Choose $\left.\varepsilon_{1}<1, \varepsilon_{2}<2 \nu(l-1)^{-2}\right)$. Using inequalities of Friedrichs for $Y_{1}, Y_{2}$, from (45), (46), we derive the inequality

$$
\begin{gathered}
\frac{d E_{2}}{d \tau}+2 \delta_{1} E_{2} \leqslant F(\tau) \\
F(\tau)=\frac{1}{2 \varepsilon_{1}} \int_{0}^{1} F_{1 \tau}^{2} d z+\frac{\rho}{2 \varepsilon_{2}} \int_{1}^{l} F_{2 \tau}^{2} d z+\frac{1}{2} F_{3 \tau}^{2}, \\
\delta_{1}=\min \left[1-\varepsilon_{1} ; \frac{2 \nu}{(l-1)^{2}}-\varepsilon_{2}\right]
\end{gathered}
$$

We obtain the estimate

$$
\begin{gathered}
E_{2}(\tau) \leqslant\left[E_{2}(0)+\int_{0}^{\tau} F(t) e^{2 \delta_{1} t} d t\right] e^{-2 \delta_{1} \tau} \\
E_{2}(0)=\frac{1}{2} \int_{0}^{1}\left(Y_{1}^{0}(z)\right)^{2} d z+\frac{\rho}{2} \int_{1}^{l}\left(Y_{2}^{0}(z)\right)^{2} d z \\
-489-
\end{gathered}
$$


with the functions $Y_{j}^{0}(z)$ from (42), (43). Hence

$$
\int_{0}^{1} U_{1 \tau}^{2}(z, \tau) d z \leqslant 2 E_{2}(\tau), \quad \int_{1}^{l} U_{2 \tau}^{2}(z, \tau) d z \leqslant \frac{2}{\rho} E_{2}(\tau) .
$$

We obtain the boundedness of the norms $U_{j \tau}$ on its domains of definition with respect to $\eta$ for all $\tau \in[0, T]$ by the estimates (20), (21), the properties of the functions $H_{1}(\eta, \tau)(23), F_{1}(\eta, \tau)$, $F_{2}(\eta, \tau), F_{3}(\tau)(31)$, inequalities $(50)$.

We multiply equation (25) by $\eta-\eta^{2}$ and integrate from 0 to 1 , after some transformations we find

$$
R_{1}(\tau)=6\left[U_{1}(1, \tau)-\int_{0}^{1}\left(z-z^{2}\right) U_{1 \tau} d z+\int_{0}^{1}\left(z-z^{2}\right) F_{1} d z\right] .
$$

Then we multiply equation (26) by $z^{2}-(l+1) z+l$ and integrate from 1 to $l$, we find

$$
R_{2}(\tau)=\frac{6}{(1-l)^{3}}\left[\nu(1-l) U_{2}(1, \tau)-\int_{1}^{l} z(z-l-1) U_{2 \tau} d z+\int_{1}^{l}\left[z^{2}-(l+1) z+l\right] F_{2} d z\right] .
$$

All the terms in the pair parts (51) and (52) are bounded for all $\tau \in[0, T]$. This follows from the obtained estimates $\left|U_{j}(\eta, \tau)\right|,\left|A_{j}(\eta, \tau)\right|,|H(\eta, \tau)|$ and their derivatives with respect to $\tau$.

\section{The asymptotic behavior of the solution for $\tau \rightarrow \infty$}

The problem (6)-(7), (25), (26), (28)-(30) has a stationary solution [7]; we denote it by $A_{j}^{s}(\eta), H^{s}(\eta), U_{j}^{s}(\eta), R_{j}^{s}$. It corresponds to the boundary data $A_{j 0}^{s}=$ const, where $A_{j}^{s}(\eta), H^{s}(\eta)$ are linear, $U_{j}^{s}(\eta)$ are polynomials of the fourth order, and $R_{j}^{s}$ are constants. Suppose that the functions $A_{j 0}(\tau)$ are defined and continuous with the derivatives $A_{j 0}^{\prime}(\tau), A_{j 0}^{\prime}(\tau)$ for all $\tau \geqslant 0$. We obtain the conditions under which the solution of the nonstationary problem for $\mathrm{y}=0$ tends to a stationary solution and establishes estimates of the rate of convergence with the help of the obtained a priori estimates $A_{j}, A_{j \tau}, U_{j}, U_{j \tau}$ and formulas (23), (24), (51), (52). To this end, we introduce the differences

$$
\begin{gathered}
N_{j}(\eta, \tau)=A_{j}(\eta, \tau)-A_{j}^{s}(\eta), \quad M(\eta, \tau)=H(\eta, \tau)-H^{s}(\eta), \\
K_{j}(\eta, \tau)=U_{j}(\eta, \tau)-U_{j}^{s}(\eta), \quad L_{j}(\tau)=R_{j}(\tau)-R_{j}^{s} .
\end{gathered}
$$

The functions $N_{j}$ is the solution of the conjugate problem (6)-(8) with $A_{10}(\tau)$ replaced by $N_{10}(\tau)=A_{10}(\tau)-A_{10}^{s}, A_{20}(\tau)$ into $N_{20}(\tau)=A_{20}(\tau)-A_{20}^{s}$, and $A_{j}^{0}(\eta)$ on $N_{j}^{0}(\eta)=A_{j}^{0}(\eta)-A_{j}^{s}(\eta)$. Therefore estimates of the form (20) are valid for

$$
\left|N_{j}(\eta, \tau)\right| \leqslant\left|N_{10}(\tau)\right|+\left(8 \varepsilon_{j} W(\tau) F(\tau)\right)^{1 / 4},
$$

with $\varepsilon_{1}=\left(\operatorname{Pr}_{1}\right)^{-1}, \varepsilon_{2}=\left(k^{2} P r_{2}\right)^{-1}$. Using the simple inequality $(a+b)^{2} \leqslant 2\left(a^{2}+b^{2}\right)$, inequality (18) and the definition of $G(\tau)$ from (17), we obtain the estimate of

$$
W(\tau) \leqslant\left(\sqrt{W_{0}}+\gamma_{1} \int_{0}^{\tau}\left[\left|N_{10}(t)\right|+\left|N_{20}(t)\right|+\left|N_{10}^{\prime}\right|+\left|N_{20}^{\prime}(t)\right|\right] e^{\delta t} d t\right) e^{-2 \delta \tau},
$$

where (см.(13), (18))

$$
\begin{gathered}
W_{0}=\frac{P r_{1}}{2} \int_{0}^{1}\left[N_{1}^{0}(\eta)-N_{10}(0)(\eta-1)^{2}\right]^{2} d \eta+\frac{k}{2 \nu} P r_{2} \int_{1}^{l}\left[N_{2}^{0}(\eta)-N_{20}(0) \frac{(\eta-1)^{2}}{(l-1)^{2}}\right] d \eta, \\
\gamma_{1}=\max \left[\frac{2}{\sqrt{P r_{1}}}, \sqrt{\frac{P r_{1}}{5}}, \frac{2}{\sqrt{k^{-1} \nu^{3}(l-1)^{3} P r_{2}}},(l-1) \sqrt{\frac{k}{2 \nu} P r_{2}}\right] .
\end{gathered}
$$


Therefore, if the integrals

$$
\int_{0}^{\infty}\left|N_{j 0}(t)\right| e^{\delta t} d t=\int_{0}^{\infty}\left|A_{j 0}(t)-A_{j 0}^{s}\right| e^{\delta t} d t, \quad \int_{0}^{\infty}\left|N_{j 0}^{\prime}(t)\right| e^{\delta t} d t=\int_{0}^{\infty}\left|A_{j 0}^{\prime}(t)\right| e^{\delta t} d t
$$

converge, then for all $\tau \geqslant 0$

$$
W(\tau) \leqslant c_{1} e^{-2 \delta \tau}, \quad c_{1}=\mathrm{const}
$$

Further, all constants of the form (56) will be denoted by $c_{2}, c_{3}, \ldots$.

For the function $F(\tau)$ from (19) we have the estimate

$$
|F(\tau)| \leqslant c_{2}+c_{3} \int_{0}^{\tau}\left[N_{10}^{2}(t)+N_{20}^{2}(t)+\left(N_{10}^{\prime}(t)\right)^{2}+\left(N_{20}^{\prime}(t)\right)^{2}\right] d t .
$$

Since the integrals $(57)$ converge, then $\left|A_{j 0}(\tau)-A_{j 0}^{s}\right| \leqslant h_{j}(\tau) e^{-\delta \tau}, \quad\left|A_{j 0}^{\prime}(\tau)\right| \leqslant \rho_{j}(\tau) e^{-\delta \tau}$ with nonnegative functions $h_{j}(\tau), \rho_{j}(\tau) \rightarrow 0$ by $\tau \rightarrow \infty$ and

$$
\int_{0}^{\infty} h_{j}(\tau) d \tau \leqslant \infty, \quad \int_{0}^{\infty} \rho_{j}(\tau) d \tau \leqslant \infty
$$

The boundedness of $|F(\tau)| \leqslant c_{4}$ follows from (59) for all $\tau \geqslant 0$. Considering (53), (54), (58) and (59) we find

$$
\left|N_{j}(\eta, \tau)\right|=\left|A_{j}(\eta, \tau)-A_{j}^{s}(\eta)\right| \leqslant c_{4+j} e^{-\delta \tau / 2}
$$

for all $\eta \in[0,1],(j=1), \eta \in[1, l],(j=2)$ and $\tau \geqslant 0$. From inequalities (21) we obtain

$$
\left|N_{j \tau}(\eta, \tau)\right|=\left|A_{j \tau}(\eta, \tau)\right| \leqslant c_{5+j} e^{-\delta \tau / 2} .
$$

Next we proceed to obtain estimate $M(\eta, \tau)=H(\eta, \tau)-H^{s}(\eta)$ from (53). First we note that the mean value of $H^{0}(\eta)$ can be considered zero:

$$
\int_{0}^{1} H_{1}^{0}(\xi) d \xi=0
$$

Given this assumption, $M$ represent from (23) and (24) as follows:

$$
\begin{gathered}
M(\eta, \tau)=\psi N_{1}(\eta, \tau)+\int_{0}^{1}\left[H^{0}(\xi)+H^{s}(\eta)-\psi A_{1}^{0}(\xi)\right] \bar{G}(\eta, \xi, \tau) d \xi- \\
-\psi \int_{0}^{1} N_{1}(\xi, \tau) d \xi-\psi \int_{0}^{\tau} \int_{0}^{1} N_{1 \mu}(\xi, \mu) \bar{G}(\eta, \xi, \tau-\mu) d \xi d \mu
\end{gathered}
$$

where $\bar{G}(\eta, \xi, \tau)=G(\eta, \xi, \tau)-1, G(\eta, \xi, \tau)$ is given by (24). Using inequalities (60), (61) for $j=1$, from (62) we obtain the estimate

$$
|M(\eta, \tau)|=\left|H(\eta, \tau)-H^{s}(\eta)\right| \leqslant c_{g} e^{-\omega_{1} \tau}, \quad \omega_{1}=\min \left(\frac{\delta}{2}, \frac{\pi^{2}}{S}\right) .
$$

In deriving (63) we took into account the inequalities

$$
n^{2 \alpha_{1}} e^{-n^{2} \alpha_{2} \varepsilon}<\frac{C^{1}\left(\alpha_{1}, \alpha_{2}, \varepsilon\right)}{n^{2}}, \quad t^{\alpha_{3}} e^{-\alpha_{4} t} \leqslant C^{2}\left(\alpha_{3}, \alpha_{4}\right)
$$


for $\alpha_{k}>0, k=1, \ldots, 4, C^{1}, C^{2}$ are bounded constants, $t \geqslant \varepsilon>0$. The estimate of $M_{\tau}$ is found from equality

$$
\begin{aligned}
M_{\tau}= & \frac{1}{S} \int_{0}^{1}\left[H^{0}(\xi)-H^{s}(\xi)-\psi A_{1}^{0}(\xi)\right] \bar{G}_{\eta \eta}(\eta, \xi, \tau) d \xi- \\
& -\frac{\psi}{S} \int_{0}^{\tau} \int_{0}^{1} N_{1 \mu}(\xi, \mu) \bar{G}_{\eta \eta}(\eta, \xi, \tau-\mu) d \xi d \mu
\end{aligned}
$$

It follows from an equation of the form (22) on $M$ and formula $(62)$, then $\left|M_{\tau}(\eta, \tau)\right|=$ $=\left|H_{\tau}(\eta, \tau)\right| \leqslant c_{10} e^{-\omega_{1} \tau}$.

We proceed to estimate the functions estimated $K_{j}(\eta, \tau), K_{j \tau}(\eta, \tau)$ from (53). We have the inequality (39) for $K_{1}$, where $F_{3}(\tau)=-2 N_{1}(1, \tau)-2 \omega M(1, \tau)$, the function $E_{1}(\tau)$ satisfies the inequality (36), and $Q(\tau)$ is determined from (38). In view of $(60),(61),(63)$, (64) we obtain

$$
\left|K_{1}(\eta, \tau)\right|=\left|U_{1}(\eta, \tau)-U_{1}^{s}(\eta)\right| \leqslant c_{10} e^{-\omega_{2} \tau}, \quad \omega_{2}=\min \left(\omega_{1}, \delta_{1}\right)
$$

Here $\delta_{1}$ is defined by the equality (48). Similarly, from (32) we obtain $\left|K_{2}(\eta, \tau)\right|=\mid U_{2}(\eta, \tau)-$ $-U_{2}^{s}(\eta) \mid \leqslant c_{11} e^{-\delta_{1} \tau}$, and from (49), (50) we derive estimates $\left|K_{j \tau}(\eta, \tau)\right|=\left|U_{j \tau}(\eta, \tau)\right| \leqslant$ $\leqslant c_{11+j} e^{-\delta_{1} \tau}$ provided that condition (57) is satisfied and the integral

$$
\int_{0}^{\infty}\left|N_{j 0}^{\prime \prime}(t)\right| e^{\delta t} d t=\int_{0}^{\infty}\left|A_{j 0}^{\prime \prime}(t)\right| e^{\delta t} d t .
$$

is converges. Thus, if convergence of the integrals (57), (65) takes place, then the nonstationary solution converges to the stationary one in accordance with the exponential law. The same applies to the functions $R_{j}(\tau)$, which defined by formulas (51), (52).

The work received financial support from RFBR (project 17-01-00229).

\section{References}

[1] A.Nepomnyashii, I.Simanovskii, J.-C.Legros, Interfacial Convection in Multilayer System, Springer, New York, 2006.

[2] R.Narayanan, D.Schwabe, Interfacial Fluid Dynamics and Transport Processes. SpringerVerlag, Berlin, 2003.

[3] R.Kh.Zeytovnian, Convection in Fluids, Springer, Dordrecht, 2009.

[4] V.K.Andreev, V.E.Zakhvataev, E.A.Ryabitskii, Thermocapillary Instability, Nauka, Novosibirsk, 2000 (in Russian).

[5] V.K.Andreev, On Inequalities of the Friedrichs type for Combined Domains, Journal Siberian Federal University. Mathematics and Physics, 2(2009), no. 2, 146-157 (in Russian).

[6] A.D.Polianin, Handbook of linear partial differential equations for engineers and scientists, Boca Raton, London, 2002.

[7] M.V.Efimova, On one two-dimensional stationary flow of a binary mixture and viscous fluid in a plane layer, Journal Siberian Federal University. Mathematics and Physics, 9(2016), no. $1,30-36$. 


\section{Априорные оценки сопряженной задачи, описывающей совместное движение жидкости и бинарной смеси \\ в канале}

\section{Виктор К. Андреев \\ Марина В. Ефимова}

Институт вычислительного моделирования СО РАН

Академгородок, 50/44, Красноярск, 660036

Сибирский федеральный университет Свободный, 79, Красноярск, 660041

Россия

Для линейной сопряженной начально-краевой обратной задачи, описывающей совместное движение бинарной смеси и вязкой теплопроводной жидкости в плоском канале, получены априорные оченки решения в равномерной метрике. C их помощъю установлено, что решение нестационарной задачи с ростом времени стремится $к$ стационарному решению по экспоненциалъному закону, если температура на стенках канала стабилизируется со временем.

Ключевые слова: сопряженная задача, обратная задача, априорные оченки, асимтотическое поведение. 